
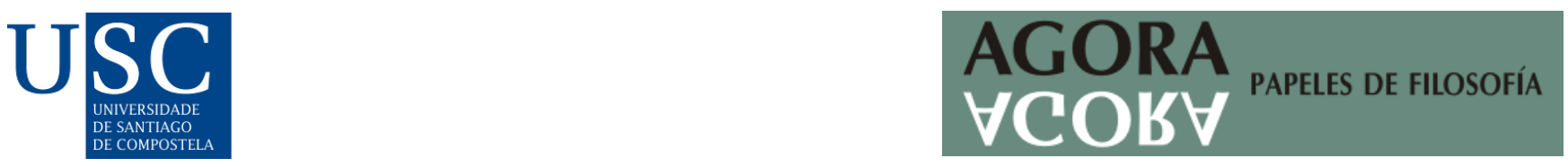

\title{
ALTRUISMO EFICAZ Y APLICACIÓN DE PRINCIPIOS ÉTICOS: UNA OPORTUNIDAD PARA LA EDUCACIÓN PARA LA CIUDADANÍA GLOBAL EN LA UNIVERSIDAD
}

\author{
Xosé Luís Pastoriza Rozas ${ }^{1}$ iD \\ ${ }^{1}$ Universidade de Santiago de Compostela, España
}

Recibido: 15/11/2020; Aceptado: 09/03/2021

\section{Resumen}

Este artículo analiza la idoneidad del altruismo eficaz como marco ético de referencia para las prácticas de educación para la ciudadanía global en la Universidad. El altruismo eficaz demanda que las prácticas altruistas y solidarias se basen no solo en decisiones emocionales, sino en un análisis racional que permita conseguir el mayor impacto posible en beneficio de terceros. Simultáneamente, las Universidades promueven la educación para la ciudadanía global que incluye el desarrollo de competencias para la aplicación de principios éticos orientados a la mejora del mundo tal y como es definida en la Agenda 2030 de Desarrollo Sostenible de Naciones Unidas. Pero la amplitud de los problemas de desarrollo requiere criterios de decisión para seleccionar aquellas causas que pueden tener un mayor y mejor impacto. Por ello, el altruismo eficaz ofrece un repertorio razonado de criterios de decisión facilitadores de la aplicación de principios éticos entre la ciudadanía global universitaria.

Palabras clave: altruismo eficaz; ética del desarrollo; ciudadanía global; ética aplicada; filosofía de la educación

\begin{abstract}
This paper analyses the suitability of Effective Altruism as an ethical frame for the practices of Education for Global Citizenship in the University. According to Effective Altruism, altruistic and solidary practices should be based not only on emotional decisions, but also on a rational analysis to achieve the greatest possible impact for the benefit of third parties. Simultaneously, Universities promote Education for Global Citizenship that includes the development of competencies for the application of ethical principles focused on the improvement of the world as defined in the United Nations 2030 Agenda for Sustainable Development. But the breadth of development problems demands decision criteria to select those causes that can have a greater and better impact. Therefore, Effective Altruism enumerates a reasoned repertoire of decision criteria that could facilitate the application of ethical principles among university global citizenship.
\end{abstract}

Keywords: effective altruism; development Ethics; global citizenship; applied Ethics; Philosophy of Education 


\section{INTRODUCCIÓN}

Este artículo describe las bases teóricas del altruismo eficaz, así como las críticas a sus planteamientos, con el fin de evaluar su idoneidad como marco ético de referencia para las prácticas de educación para la ciudadanía global en las Universidades españolas. Los partidarios del altruismo eficaz se organizan en comunidades locales y globales de reflexión y práctica declarando su intención de hacer el máximo bien en el mundo, pero en España su relevancia pública aún resulta limitada tanto a nivel académico como de la sociedad civil ${ }^{1}$. Al mismo tiempo, la reflexión teórica y las experiencias de educación para la ciudanía global integran una dimensión normativa, aunque no siempre se profundice en su fundamentación más allá de meras declaraciones de buenas intenciones. Por este motivo, resultaría deseable establecer conexiones entre los activistas del altruismo eficaz y los promotores y practicantes de la educación para la ciudanía global en la Universidad de tal modo que se constituya una comunidad de diálogo que permita superar algunas de las limitaciones que se identifican en el ámbito de la educación para la ciudadanía global tanto en la aplicación de principios éticos como en la toma de decisiones respecto de la selección de las causas sociales con más y mejor impacto en el bienestar de terceros.

Aunque el concepto de ciudanía global constituye un objeto de controversia y diversidad de interpretaciones (Agra, 2002; Cabrera, 2008), la UNESCO describe la ciudadanía global como el desarrollo de un sentimiento de pertenencia a una comunidad ampliada y a una humanidad común junto con la conciencia de la interdependencia e interconexión entre lo local, lo nacional y lo global (UNESCO, 2014). Para el desarrollo de este sentimiento y conciencia, resulta posible diseñar y ejecutar procesos de enseñanza-aprendizaje específicos que empoderen a las personas educandas para que asuman un rol activo en la construcción de sociedades más pacíficas, tolerantes, inclusivas, sostenibles y seguras. Estos procesos educativos, con pluralidad de metodologías y enfoques, se agrupan bajo la denominación genérica de educación para la ciudadanía global.

Las Universidades constituyen un agente activo en la sensibilización y movilización de la comunidad universitaria en relación con los problemas de desarrollo, tal y como aparecen descritos en la Agenda 2030 de Desarrollo Sostenible de las Naciones Unidas (Naciones Unidas, 2015). Al mismo tiempo promueven la construcción de una ciudadanía global solidaria y comprometida con la transformación social positiva en el marco de la vigente agenda global de desarrollo. Sin embargo, el hecho de que esta agenda se traduzca operativamente en 17 Objetivos de Desarrollo Sostenible (ODS) y 169 metas sugiere la necesidad de desarrollar procesos de decisión individuales, grupales y comunitarios para seleccionar aquellas causas que pueden tener un mayor y mejor impacto. Porque, aunque la Agenda 2030 enfatiza la universalidad, integralidad e indivisibilidad de sus objetivos y metas no resulta sensato pensar que los actores individuales puedan actuar en la totalidad de los problemas de desarrollo definidos por la misma. Por tanto, para evitar que las responsabilidades compartidas pero diferenciadas a las que apela la Agenda 2030 se conviertan en deberes superogatorios, consideramos necesario definir criterios de decisión que orienten las capacidades limitadas y recursos escasos de los que disponen los individuos hacia las causas con mayor y mejor impacto en el bienestar de terceros.

El altruismo eficaz como movimiento social ha hecho su aparición en el escenario global demandando que las prácticas altruistas y solidarias se basen no solo en decisiones emocionales, sino en un análisis racional que permita conseguir el mayor impacto posible en beneficio de terceros, sean o no humanos. Por este motivo, sus activistas ofrecen una combinación de varios 
criterios de decisión como la escala, desatención y tratabilidad de los problemas que facilitan la selección de aquellas causas sociales con un mayor y mejor impacto. La apelación a un análisis racional motiva una preferencia por el uso de evidencias acerca de cómo son las cosas, cuál es el alcance de los problemas que afectan al bienestar y calidad de vida de terceros y qué impactos tienen nuestras acciones individuales y colectivas en su paliación. Además, promueve el uso de métodos de reflexión rigurosos con una doble finalidad. En primer lugar, para poder concluir de qué modo maximizar aquello que es valioso dados ciertos recursos limitados a nuestra disposición, entendiendo aquello que es valioso de forma imparcial y conforme a una posición bienestarista que de forma tentativa afirma que existen razones para actuar en beneficio de terceros y promover su bienestar en el mayor grado posible (MacAskill y Pummer, 2020). Y, en segundo lugar, para intentar mejorar el mundo en función de los resultados de tal reflexión. De esta forma, el altruismo eficaz ofrecería un marco idóneo para la promoción de la ciudadanía global entre la comunidad universitaria ya que facilita criterios para orientar las decisiones y acciones individuales y colectivas centradas en mitigar y superar los problemas globales de desarrollo enumerados en la mencionada Agenda 2030.

En primer lugar, analizamos el reconocimiento y legitimidad de las Universidades como espacio educativo orientado a la construcción de ciudadanía global. En segundo lugar, identificamos un espacio desatendido de aprendizaje en las Universidades vinculado al desarrollo de la competencia para la aplicación de principios éticos. Por último, revisamos la propuesta del altruismo eficaz como un fármaco y remedio para la aplicación efectiva y racional de principios éticos para la mejora del mundo, de tal modo que pueda orientar las decisiones éticas del estudiantado universitario en relación con las causas sociales que deciden apoyar, la elección de carrera profesional y la práctica de las virtudes asociadas a una vida buena.

\section{LAS UNIVERSIDADES COMO AGENTES DE CONSTRUCCIÓN DE CIUDADANÍA GLOBAL}

En los últimos veinte años, las Universidades han sido reconocidas como actores centrales del sistema español de cooperación a nivel estatal y autonómico, un proceso paralelo al desarrollo del Espacio Europeo de Educación Superior y a la reflexión en torno a la renovación de la misión de las instituciones de educación superior. En este doble proceso, la cooperación universitaria para el desarrollo opera como un ámbito de especialización institucional que materializa la aspiración colectiva de una Universidad emprendedora, innovadora y socialmente comprometida que contribuya a la realización de la agenda global de desarrollo sostenible que en la actualidad se articula a través de la Agenda 2030 de Desarrollo Sostenible de las Naciones Unidas y sus 17 Objetivos de Desarrollo Sostenible (ODS) (CRUE, 2000, 2019).

Con el fin de promover la consecución de la Agenda 2030, el Gobierno de España aprobó en 2018 el Plan de Acción para la Implementación de la Agenda 2030 Hacia una Estrategia Española de Desarrollo Sostenible (Gobierno de España, 2018), donde se recoge el posicionamiento de la Conferencia de Rectores de las Universidades Españolas (CRUE) y se enumeran las contribuciones específicas que las Universidades pueden aportar a la aplicación efectiva de la Agenda 2030. Entre ellas se destaca el «compromiso decidido con la inclusión de competencias relacionadas con un desarrollo sostenible e inclusivo, necesarias para la construcción de una ciudadanía global, en la formación de todo el estudiantado, el personal docente e investigador y el personal de 
administración y servicios» (p. 128). Este punto se alinea con el ODS 4 (Garantizar una educación inclusiva, equitativa y de calidad y promover oportunidades de aprendizaje durante toda la vida para todas las personas) y su meta 4.7 centrada en la educación para el desarrollo sostenible o educación para la ciudanía global.

Progresivamente, las Universidades españolas han asumido su responsabilidad en la promoción del desarrollo humano sostenible tal y como lo define el PNUD en su Informe de Desarrollo Humano del año 1994: «El desarrollo humano sostenible es el desarrollo que no sólo suscita un crecimiento económico, sino que también distribuye equitativamente sus beneficios; que regenera el medio ambiente en lugar de destruirlo; que fomenta la autonomía de las personas en lugar de marginarlas» (p. iii). Además, han incorporado institucionalmente no solo sus principios y valores, sino que han implementado metodologías de educación transformadora, de aprendizaje activo, reflexivo, participativo y experiencial para el desarrollo de las competencias necesarias para que el conjunto de la comunidad universitaria se dote de una «comprensión crítica de la problemática social, económica y ambiental, global y local, la aplicación de procedimientos para la toma de decisiones y realización de acciones coherentes con la Agenda 2030» (Gobierno de España, 2018, p. 128). Por tanto, las Universidades aspiran a construir una ciudadanía capacitada para la transformación social positiva a través del fomento de aquellos conocimientos, habilidades, actitudes y valores necesarios para afrontar los retos actuales y futuros de la Humanidad y del Planeta (CRUE, 2012).

No obstante, resulta necesario analizar más en detalle cómo se operativizan los diversos valores e imperativos éticos a la hora de tomar decisiones relativas a cómo mejorar el mundo y cómo desarrollar una vida personal y profesional coherente con dichos valores y principios. En este sentido, creemos que la «competencia en la aplicación de principios éticos relacionados con los valores de la sostenibilidad en los comportamientos personales y profesionales» (CRUE, 2012, p. 7) apenas ha recibido una atención específica más allá de declaraciones genéricas. Intuimos que esta elusión y olvido se debe al esfuerzo por mantener un cierto grado de neutralidad ética en las Universidades públicas que las disuade de prescribir determinados modelos o proyectos de vida buena que implican un determinado proceso de toma de decisiones éticas.

En nuestra opinión, las Universidades deben cultivar la humanidad fomentando un aprendizaje ético que construya ciudadanía y teja lazos solidarios entre lo local y lo global, entre lo propio y lo ajeno, entre lo próximo y lo alejado, entre la vida humana y no humana para afrontar el futuro de la vida en común. Para este cultivo de la Humanidad, la filósofa Martha C. Nussbaum (1998) nos prescribe tres fármacos: en primer lugar, un autoexamen crítico, consciente y deliberativo de nuestras creencias y capacidades con el fin de ver con mayor claridad nuestro presente y anticipar el futuro; en segundo lugar, la actuación como ciudadanos del mundo o ciudadanos globales donde nos definimos y actuamos en función de aspiraciones y preocupaciones más universales con un deseo de amistad, hospitalidad y amor por toda la humanidad; y, en tercer lugar, la construcción individual y colectiva de un relato de igualdad, inclusión, distribución y justicia global que nos dote de una identidad en la que nos reconozcamos como miembros de una misma comunidad planetaria, con raíces e identidades locales, y con deberes de cooperación y ayuda mutua. Tres fármacos que, desnudados de su abstracción, sería posible identificar en las personas que apuestan por convicción por la solidaridad local con proyección global en las instituciones de Educación Superior. 


\section{LAS COMPETENCIAS PARA LA CIUDADANÍA GLOBAL Y LA APLICACIÓN DE PRINCIPIOS ÉTICOS: UN ESPACIO DESATENDIDO DE APRENDIZAJE}

Antes de analizar las diferentes propuestas que están impulsando la educación para la ciudadanía global a nivel europeo e internacional, queremos señalar que el debate sobre la necesidad de fomentar la ciudadanía global parte de una serie de evidencias:

- La tragedia que representa la indiferencia de las sociedades opulentas y de las élites de los países empobrecidos respecto de la pobreza extrema y de la desigualdad, indiferencia que perpetúa la exclusión y la falta de oportunidades para millones de personas en el mundo (Pogge, 2005). Además, a esta tragedia habría que añadir la indiferencia ante la crisis ecológica y las vidas de los animales no humanos.

- La existencia de un colectivo amplio de personas que se definen a sí mismas como ciudadanas globales (Schattle, 2008).

- La convivencia de un conjunto plural de imaginarios de ciudadanía global que abarcan desde la imagen cosmopolita clásica del viajero trotamundos hasta el activista altermundialista, pasando por la élite financiera de la globalización y los integrantes de las organizaciones supranacionales gubernamentales y no gubernamentales (Falk, 1994).

Por otra parte, las diferentes definiciones disponibles de educación para la ciudadanía global coinciden en echar raíces en una filosofía cosmopolita, humanista y republicana que centramos en cinco afirmaciones que responden a la doble cuestión de qué tipo de ciudadano se aspira a formar y qué clase de ciudadanía se debe promover desde las Universidades (Pastoriza, 2014):

- La ciudadanía se adquiere en la práctica y como rol a través del ejercicio de las virtudes cívicas y de los deberes ampliados hacia la humanidad en su conjunto.

- En la práctica cívica cotidiana se deben materializar los valores de la participación, igualdad, solidaridad, fraternidad, equidad, tolerancia y justicia social, siendo conscientes de los desacuerdos existentes sobre la naturaleza de dichos valores y sus implicaciones prácticas.

- Se debe promover una comunidad imaginada de ciudadanos del mundo y una identidad cosmopolita como reconocimiento de la interdependencia y de la común pertenencia a la humanidad.

- La ciudadanía tiene responsabilidades en la construcción de un mundo más justo a nivel local y global.

- Toda transformación del sistema global abarca un campo ético (ética global) e institucional (gobernanza global) que va asociado dialécticamente a un proceso de cambio personal y a la toma de decisiones éticas en la vida cotidiana.

Por tanto, la educación para la ciudadanía global se aleja de cualquier pretensión de formar "santos morales" (Wolf, 1982) pero actualiza la misión de la filosofía política y de la ética de promover «una vida buena, con y para los otros, en instituciones justas» (Ricoeur, 2006) para que cada persona tenga la oportunidad de actuar como un ciudadano global informado, responsable y comprometido. Desde nuestro punto de vista, la educación para la ciudadanía global complementa 
esta misión en un doble nivel: en primer lugar, integrando una visión cosmopolita; y, en segundo lugar, apostando por la configuración de instituciones liberadoras que reconozcan la prioridad ética y política en la construcción del espacio público local y global de la visión y la voz de las víctimas o de las personas que sufren más intensamente (Dussel, 1998). Desde este punto de vista, la educación para la ciudadanía global debe de apostar por una educación crítica que aborde con radicalidad las desigualdades de poder y voz, y que complemente el aprendizaje crítico con el aprendizaje cívico. Aspira, por tanto, a empoderar a cada persona para que adquiera la capacidad de construir una lectura del mundo por sí misma, sea capaz de hacer escuchar su voz, y sea capaz de tomar decisiones éticas comprometidas con el cambio social (Andreotti, 2006).

En los últimos años, se han ido configurando una serie de propuestas alineadas con la promoción de la educación para la ciudadanía global, la educación para la paz o la educación para el desarrollo sostenible tanto desde el ámbito académico como desde el institucional, entre los que destacamos el marco de competencia global del Estudio Pisa de la OCDE (2018), la educación para la ciudadanía global y la educación para los Objetivos de Desarrollo Sostenible de la UNESCO (2015 y 2017) o el marco de competencias para una cultura democrática del Consejo de Europa (2018). Estas propuestas integran un conjunto común de competencias y dimensiones (conocimientos, actitudes, valores y habilidades) que se pueden sintetizar en: conocimiento de temas y problemas globales; capacidad para el pensamiento crítico; comprensión y sensibilidad hacia culturas diferentes; empatía y solidaridad hacia otras culturas y sociedades; respeto a la diversidad, las diferencias y otros valores; sentido de pertenencia a la humanidad común; capacidad para la comunicación intercultural; actitud de responsabilidad social; capacidad para resolver cooperativa y responsablemente conflictos y problemas; voluntad de proteger el medio ambiente y los derechos humanos; participación activa y eficaz en la comunidad local y global; actitud para preservar la paz; actitud hacia la justicia global y la equidad; y conocimiento de uno mismo (GacelÁvila, 2017).

En todas estas propuestas se integra una dimensión ética vinculada a actitudes y valores como el respeto a la vida y la dignidad humana, a la diversidad cultural, la igualdad de derechos, la equidad y la justicia social, el desarrollo de un sentido de solidaridad humana y responsabilidad compartida y de actitudes de cuidado, altruismo y empatía. Esta dimensión ética también incluye el tomar decisiones de acción con base en principios éticos en favor del bienestar colectivo y del desarrollo sostenible que contribuyan a la resolución de los problemas globales y a la consecución del bien común y la justicia global. No obstante, es posible identificar en dichas propuestas la limitada disponibilidad de orientaciones para la comunidad universitaria respecto de cómo desarrollar la aplicación efectiva de principios éticos para la mejora del mundo. Es decir, la deseable promoción del cuidado, el altruismo y la empatía se limita en la mayoría de los casos a una mera declaración de intenciones que no define criterios precisos respecto de la toma de decisiones éticas en favor del bienestar colectivo. Esta desatención es la que creemos que supera la propuesta del altruismo eficaz.

\section{ALTRUISMO EFICAZ: UN FÁRMACO PARA LA APLICACIÓN EFECTIVA Y RACIONAL DE PRINCIPIOS ÉTICOS PARA LA MEJORA DEL MUNDO}

En la actualidad se está desarrollando un nuevo movimiento social que aborda la reflexión filosófica sobre la práctica ética en la vida cotidiana: el altruismo eficaz. Aunque se reconoce su 
vinculación intelectual con el utilitarismo, no todos los partidarios del altruismo eficaz se declaran utilitaristas y únicamente afirman que ayudar a terceros es relevante e importante al tiempo que centran su atención en cuestiones no exclusivamente ligadas al bienestar como la desigualdad o la realización de los derechos humanos, entre otras (McMahan, 2016). Por otra parte, a pesar de que se haya puesto en cuestión experimentalmente que el contacto con la ética, al menos en su dimensión educativa o académica, mejore la condición moral de las personas (Schwitzgebel, Cokelet y Singer, 2020), los proponentes del altruismo eficaz comparten la convicción de que el contacto con la ética debe mejorar la vida de las personas. Su punto de partida es sencillo: deberíamos hacer todo lo que esté en nuestras manos para mejorar el mundo y ayudar a los demás tanto como sea posible. Esta dimensión propia del altruismo en su dimensión emocional se combina con una exigencia de racionalidad y evidencia con el fin de conseguir el mayor impacto y eficacia en el uso de unos recursos personales limitados que ponemos a disposición del bienestar y beneficio de terceros en el mayor grado posible. Por tanto, el altruismo eficaz sugiere la necesidad del paso de una solidaridad meramente empática o sentimentalista a una solidaridad racional que nos afirme en nuestra creencia de que debemos conseguir un gran bien para el mayor número posible o que el bien de las otras personas es tan importante como nuestro propio bien. En síntesis, se trata de «hacer todo el bien que se pueda» de una manera imparcial (MacAskill, 2015; Todd, 2016; Singer, 2017; Vinding, 2018).

Para ello, el altruismo eficaz impone la exigencia de llevar una vida examinada y reflexiva que analice si mis acciones contribuyen a lograr el máximo bien posible a terceros a lo largo de mi vida, partiendo de la base de que el bienestar de los demás me preocupa y que estoy dispuesto a introducir cambios sustanciales en mi vida para mejorar el mundo. Esta idea de mejorar el mundo aparece como una creencia de sentido común asumida como parte integrante de la ética de mínimos exigible, en principio, a cualquier persona que quiera llevar una vida mínimamente ética y decente. Al mismo tiempo, la posibilidad de cambiar nuestra vida de forma positiva constituye una aspiración ética que debe superar los riesgos de la credulidad, el desencanto, la frustración y la desorientación. Frente a estas amenazas, el altruismo eficaz ofrece criterios para tomar decisiones éticas que nos permitan hacer el máximo bien que podamos a partir de los recursos limitados de los que disponemos. Por tanto, alimenta la posibilidad de una vida realizada, ya que ofrece un sentido y la posibilidad de encontrar satisfacción con aquello que hacemos. En este sentido, sentirse bien al hacer el bien o aumentar la felicidad personal, no es un síntoma de egoísmo, sino una señal que confirma la bondad de la acción que llevamos a cabo.

Para el altruismo ético, el fundamento más sólido de la autoestima consiste en vivir una vida en la que cada uno contribuye en la mayor medida de lo posible a hacer del mundo un sitio mejor. Por estos motivos, el altruismo eficaz no implica sacrificio, enajenación o pérdida de identidad, sino que es expresión genuina de la propia identidad ya que lo realmente importante es la preocupación que tienen las personas por los intereses de los demás, de tal modo que aumentar el bienestar ajeno nos hace felices (Singer, 2017, p. 121). Pero más allá de esta dimensión individual, el altruismo eficaz destaca el poder que los individuos tenemos en las sociedades opulentas para realizar el bien de una manera inteligente y provocar cambios a nivel micro y macro superando los obstáculos cognitivos, emocionales y políticos con los que se encuentran las decisiones de donación o los compromisos personales con el cambio social positivo.

En líneas generales, el altruismo eficaz implica una triple exigencia. En primer lugar, demanda la necesidad de superar la visión conformista que opina que llevar una vida éticamente aceptable incluye únicamente el altruismo y la solidaridad reducidos a la donación de parte de los recursos 
que nos sobran. En segundo lugar, la promoción del altruismo y la filantropía delimita una exigencia institucional: las organizaciones e instituciones beneficiarias de las posibles donaciones deben ser transparentes y mostrar su eficacia e impacto. En tercer lugar, esta corriente ética modula las decisiones fruto de las reacciones emocionales ante las situaciones de sufrimiento, catástrofe o desgracia e introduce elementos racionales para que la toma de decisiones se realice con base en datos sólidos y robustos, sea eficaz y consiga el mayor bien posible. De hecho, introduce una exigencia de imparcialidad, de tal modo que se traten equitativamente aquellos intereses iguales. Es decir, que ante dos sufrimientos iguales debemos comprometernos a paliarlos con independencia de quiénes los experimenten.

El altruismo eficaz se basa en cuatro principios éticos de raigambre bienestarista (Singer, 2017).

- La consideración de que las vidas humanas y no humanas son valiosas, aunque este valor pueda variar en función de su calidad de vida.

- El deber de amar y cuidar al prójimo cercano, pero también al prójimo alejado y distante.

- La obligación de conseguir el máximo bien para todas las personas.

- El compromiso con la mejora del mundo local y global en el que vivimos.

Estos principios éticos se conectan con la dimensión normativa de la ciudadanía global que afirma la existencia de deberes ampliados hacia la humanidad (Dower, 2003). El compromiso con la realización de dichos principios en la vida cotidiana a través de nuestros sentimientos de empatía, nuestras decisiones de generosidad, y nuestro compromiso con el bienestar presente y futuro de terceros ejemplifica cómo los seres humanos somos capaces de superar los límites de la familia, amistades, comunidades y estados sin asumir una ética de sacrificio y renuncia. Al mismo tiempo, estos principios éticos operan como antídotos frente al egoísmo, el racismo y el supremacismo de cualquier naturaleza.

El altruismo eficaz nos motiva a conseguir el máximo bien y a conseguir un mundo en el que haya menos sufrimiento y más felicidad, seleccionando aquellas causas maximizadoras del bien común incluyendo el sufrimiento humano y no humano. Su objetivo es promover la justicia, la libertad, la igualdad y el conocimiento para que las personas puedan vivir mejor, libres de opresión, con más respeto cara sí mismas y más libertad para llevar la vida que quieran (Singer, 2017). De todos modos, el altruismo eficaz nos impone la exigencia de analizar si realmente estamos haciendo lo más eficaz y sensato y si existen alternativas con un menor coste y un mayor impacto en términos de maximización del bien.

Para este análisis, se proponen una serie de criterios de decisión basados en una serie de preguntas que debemos resolver con el fin de obtener el mejor curso de acción posible a la hora de tomar una decisión relativa a la disposición de los limitados recursos personales al servicio de la maximización del bien (MacAskill, 2015; Vinding, 2018):

- ¿Cuántas personas se beneficiarán y en qué grado?

- ¿Es la opción más eficaz que se puede realizar?

- ¿Se trata de una causa o problema desatendido?

- ¿Qué habría pasado de todos modos sin nuestra intervención?

- ¿Cuáles son las probabilidades de éxito y cuánto bien se conseguirá?

A pesar de su aparente simplicidad, la experiencia muestra que existen numerosas causas y problemas sociales que demandan nuestra atención y que compiten por convertirse en objeto 
de nuestra generosidad o solidaridad, como por ejemplo los problemas globales de desarrollo identificados en la Agenda 2030 de Naciones Unidas. Para poder discernir entre causas y poder jerarquizarlas con el fin de contar con una relación de prioridades, el altruismo eficaz propone cuatro criterios de decisión para seleccionar dichas causas. Estos criterios no se preocupan por la urgencia sino por el máximo bien que podemos hacer, es decir, por el mayor impacto que podemos producir con nuestra decisión racional:

- Escala: debemos investigar sobre la magnitud y alcance del problema y en qué medida afecta a la vida de las personas a corto y largo plazo.

- Manejabilidad: es importante saber si resulta fácil realizar avances en dicho problema y si resulta sencillo medir dichos progresos. Al mismo tiempo, se trata de averiguar si ya se están realizando avances y cuál es la evidencia respecto de su impacto. Por último, debemos averiguar si existen intervenciones innovadoras con alto impacto en la causa con la que queremos colaborar.

- Desatención: antes de priorizar una causa debemos conocer cuántos recursos se están dedicando a dicho problema y en qué medida están bien asignados los recursos que lo afrontan. Además, es necesario determinar si el mercado o el sector público no son capaces de resolverlo.

- Aptitud personal: determinar en qué medida podemos realizar una contribución significativa a dicho problema teniendo en cuenta nuestras habilidades, recursos, conocimientos, pasiones y vínculos.

Sin embargo, a pesar de sus virtudes, el altruismo eficaz ha recibido críticas por su individualismo; su exceso de cuantificación más propio de una racionalidad instrumental que de una racionalidad emancipadora; el tratamiento insuficiente de las cuestiones relativas a la equidad; la consideración superficial de la multidimensionalidad y complejidad de numerosas problemáticas sociales como la pobreza; la dificultad en algunos casos para integrar un enfoque basado en derechos humanos a la hora de valorar el impacto o pertinencia de una donación; la débil atención prestada a los procesos de cambio social a medio y largo plazo promovidos por las actividades de sensibilización, concienciación, movilización e incidencia propios de la educación para la ciudadanía global; el riesgo de una visión tecnocrática y de arriba-abajo que no toma en consideración las opiniones y preferencias de las personas afectadas o que sus prácticas evadan la rendición de cuentas y transparencia propias de sociedades democráticas; y, por último, el hecho de eludir el cambio sistémico o estructural necesario para superar los principales problemas de la Humanidad y el Planeta, como la pobreza, las desigualdades y la crisis ecológica y que van más allá de la lógica de la donación e implican redistribuciones de poder más que de meros recursos económicos (Gabriel, 2016; Broi, 2019).

Todas estas críticas están siendo abordadas por los principales referentes teóricos y prácticos del altruismo eficaz con el fin de dotar de mayor coherencia y rigor a su propuesta. De todos modos, como corriente que se busca a sí misma todavía resulta prematuro evaluar el alcance de este movimiento para valorar si se trata de una moda pasajera o una propuesta sólida que puede satisfacer la demanda de criterios claros, sencillos y específicos para la aplicación de principios éticos bienestaristas de forma imparcial.

Pero más allá de dichas limitaciones, el altruismo eficaz se puede constituir en uno de los modelos para la aplicación de principios éticos que demandan las prácticas y experiencias de 
educación para la ciudadanía global en la Universidad. De hecho, a pesar de las críticas, el altruismo eficaz se está revelando, desde su reciente eclosión, como un movimiento social activo que ofrece una serie de preguntas generadoras y criterios racionales de decisión ética que pueden resultar atractivos para los estudiantes universitarios preocupados por mejorar el mundo que habitamos a partir del cambio personal y del compromiso profesional. De hecho, sus principales promotores responden al perfil de joven profesional universitario de tal modo que se puede interpretar como un movimiento que ha autogestionado intelectual y prácticamente una propuesta de superación de las limitaciones de las instituciones de Educación Superior a la hora de ofrecer el desarrollo de la deseable competencia para la aplicación de principios éticos.

\section{CONCLUSIONES}

Las Universidades españolas han declarado oficialmente su compromiso con los procesos de enseñanza-aprendizaje centrados en la competencia para la aplicación de principios éticos. Al mismo tiempo, el papel de las Universidades como agentes activos en la promoción de la educación para la ciudadanía global demanda que se preste una atención específica a la dimensión normativa de la ciudadanía global. Por otra parte, las prácticas de educación para la ciudadanía global en la Universidad asumen como marco de referencia la Agenda 2030 de Desarrollo Sostenible de las Naciones Unidas que en su meta 4.7. alienta por la extensión la educación para el desarrollo sostenible o educación para la ciudanía global. Sin embargo, la amplitud de los problemas globales sociales, económicos y medioambientales identificados en dicha agenda, evidencian la necesidad de contar con criterios razonados de decisión que orienten las capacidades limitadas y recursos escasos de los que disponen los individuos para conseguir un mayor y mejor impacto en beneficio de terceros, mejorar el mundo y hacer todo el bien que se pueda.

En este sentido, las propuestas teóricas y prácticas del altruismo eficaz se pueden considerar una oportunidad individual e institucional para dotar de un marco ético de referencia a las experiencias de ciudadanía global en la Universidad. A pesar de su aparente simplicidad el altruismo eficaz no debe ocultar la complejidad de las posiciones que defiende tal y como se revela en el debate público, abierto, plural y transparente que ha promovido desde sus inicios. De hecho, se podrá estar o no de acuerdo con sus planteamientos, formulaciones y decisiones prácticas, pero no se le puede negar su valor y utilidad como alternativa preferible a la desatención de la aplicación efectiva y racional de principios éticos para la mejora del mundo desde la educación universitaria para la ciudanía global. Por eso el reto y desafío a corto y medio plazo quizás sea fomentar un diálogo mutuo y espacios de colaboración entre las dos comunidades de práctica del altruismo eficaz y la ciudadanía global para fomentar estudiantes y egresados universitarios comprometidos, responsables, activos y con sentido de la justicia local y global.

\section{Bibliografía}

Agra Romero, María Xosé (2002). Ciudadanía: fronteras, círculos y cosmopolitismo. Anales de la Cátedra Francisco Suárez, 36, 9-28.

Andreotti, Vanessa (2006). Soft versus critical global citizenship education. Policy and Practice: $A$ Development Education Review, vol. 3, Autumn, 40-51. Disponible en: https://www.developmenteduc ationreview.com/issue/issue-3/soft-versus-critical-global-citizenship-education 
Broi, Antonin (2019). Effective Altruism and Systemic Change. Utilitas, 31(3), 262-276. https://doi.org/10 $.1017 /$ S0953820819000153

Cabrera, Luis (2008). Global Citizenship as the Completion of Cosmopolitanism. Journal of International Political Theory, 4(1), 84-104. https://doi.org/10.3366/E1755088208000104

Consejo de Europa (2018). Competencias para una cultura democrática. Convivir en pie de igualdad en sociedades democráticas culturalmente diversas. Estrasburgo: Consejo de Europa. Disponible en: https $: / /$ rm.coe.int $/ 16806 \mathrm{ccc} 0 \mathrm{~d}$

CRUE (2000). Estrategia de Cooperación Universitaria para el Desarrollo. Conferencia de Rectores de las Universidades Españolas. Disponible en: http://www.ocud.es/es/files/doc555/escude2000.pdf

CRUE (2012). Directrices para la introducción de la sostenibilidad en el curriculum. Conferencia de Rectores de las Universidades Españolas. Disponible en: https://docplayer.es/16105151-Directrices-para-la-in troduccion-de-la-sostenibilidad-en-el-curriculum-crue.html

CRUE (2019). Directrices de la cooperación universitaria al desarrollo para el período 2019-2030. Conferencia de Rectores de las Universidades Españolas. Disponible en: http://www.ocud.es/es/files/doc971/dir ectrices-de-la-cooperacion-universitaria-al-desarrollo-para-el-period.pdf

Dower, Nigel (2003). An Introduction to Global Citizenship. Edinburgh: Edinburgh University Press.

Dussel, Enrique (1998). Ética de la Liberación en la Edad de la Globalización y la Exclusión. Madrid: Trotta.

Falk, Richard (1994). The Making of Global Citizenship. En Bart Van Steenbergen (ed.), The Condition of Citizenship. London: Sage, 127-140.

Gabriel, Iason (2016). Effective Altruism and its Critics. Journal of Applied Philosophy, 34 (4), 457-473. http s://doi.org/10.1111/japp.12176

Gacel-Ávila, Jocelyne (2017). Ciudadanía global. Concepto emergente y polémico. Educación Superior y Sociedad, 21, 39-63. Disponible en: https://www.iesalc.unesco.org/ess/index.php/ess3/article/view $/ 26 / 26$

Gobierno de España (2018). Plan de Acción para la Implementación de la Agenda 2030. Hacia una Estrategia Española de Desarrollo Sostenible. Madrid: Ministerio de Asuntos Exteriores, Unión Europea y Cooperación. Disponible en: http://www.exteriores.gob.es/Portal/es/SalaDePrensa/Multimedia/Publicaciones/Documents/ PLAN\%20DE\%20ACCION\%20PARA\%20LA\%20IMPLEMENTACION\%20DE\%20LA\%20AGENDA\%20 2030.pdf

MacAskill, William (2015). Doing Good Better. How Effective Altruism Can Help You Make a Difference. New York: Gotham Books.

MacAskill, William and Pummer, Theron (2020). Effective Altruism. International Encyclopedia of Ethics. ht tps://doi.org/10.1002/9781444367072.wbiee883

McMahan, Jeff (2016). Philosophical Critiques of Effective Altruism. The Philosophers' Magazine. 73, 92-99. https://doi.org/10.5840/tpm20167379

Naciones Unidas (2015). Transformar nuestro mundo: la Agenda 2030 para el desarrollo sostenible. New York: Naciones Unidas. Disponible en: https://www.un.org/ga/search/view_doc.asp?symbol=A/RES/70/1 \&Lang=S

Nussbaum, Martha Craven (1998). El cultivo de la humanidad. Una defensa clásica de la reforma en la educación liberal. Barcelona: Paidós, 2005.

OCDE (2018). Marco de competencia global. Estudio PISA. Preparar a nuestros jóvenes para un mundo inclusivo y sostenible. Disponible en: http://www.educacionyfp.gob.es/inee/dam/jcr:df1f4128-5a8f-46f2-b0c4 -498f3eb16698/PISA\%202018\%20Marco\%20Competencia\%20Global\%20(Digital).pdf 
Pastoriza Rozas, Xosé Luís (2014). As trabes de ouro. Unha educación en man común para a cidadanía global. En Pastoriza Rozas, Xosé Luís (Ed.). Educar para a cidadanía global. Experiencias, ferramentas e discursos para o cambio social. Vigo: Fundación Isla Couto, 9-12. Disponible en: http://fundacionislacouto.org/wp-content/uploads/2014/09/Educar-para-a-cidadan\%C3\%ADaglobal_FundacionIslaCouto_2014.pdf

PNUD (1994) Informe sobre desarrollo humano. México: Programa de Naciones Unidas para el DesarrolloFondo de Cultura Económica.

Pogge, Thomas (2005). La pobreza en el mundo y los derechos humanos. Barcelona: Paidós.

Ricoeur, Paul (2006). Sí mismo como otro. México: Siglo XXI.

Schattle, Hans (2008). The Practices of Global Citizenship. Lanham: Rowman \& Littlefield Publishers.

Schwitzgebel, Eric, Cokelet, Bradford y Singer, Peter (2020). Do ethics classes influence student behavior? Case study: Teaching the ethics of eating meat. Cognition, 203 (4), https://doi.org/10.1016/j.cognitio $\underline{\text { n.2020.104397. }}$.

Singer, Peter (2017). Vivir éticamente. Cómo el altruismo eficaz nos hace mejores personas. Barcelona: Paidós. Todd, Benjamin (2016). 80,000 hours. Find a Fulfilling Career that Does Good. Oxford: Centre for Effective Altruism.

UNESCO (2014), Global citizenship education: Preparing learners for the challenges of the 21st century. Paris: UNESCO. Disponible en: https://unesdoc.unesco.org/ark:/48223/pf0000227729

UNESCO (2015). Global Citizenship Education. Topics and Learning Objectives. Paris: UNESCO. Disponible en: https://unesdoc.unesco.org/ark:/48223/pf0000232993

UNESCO (2017). Educación para los Objetivos de Desarrollo Sostenible. Objetivos de Aprendizaje. Paris: UNESCO. Disponible en: https://unesdoc.unesco.org/ark:/48223/pf0000252423

Vinding, Magnus (2018). Effective Altruism. How Can We Best Help Others? North Carolina: The Neuroethics Foundation.

Wolf, Susan (1982). Moral Saints. The Journal of Philosophy, 79(8), 419-439. doi:10.2307/2026228

Notas

$1 \quad$ En noviembre de 2019, el autor realizó una presentación breve del altruismo eficaz durante el VII Congreso Universidad y Cooperación al Desarrollo celebrado en la Universidad de Santiago de Compostela. Durante el debate, los gestores y practicantes de la cooperación universitaria para el desarrollo y la educación para la ciudadanía global presentes en su sesión afirmaron desconocer el altruismo eficaz. 